\title{
A method to represent ozone response to large changes in precursor emissions using high-order sensitivity analysis in photochemical models
}

Greg Yarwood ${ }^{1}$, Christopher Emery $^{1}$, Jaegun Jung ${ }^{1}$, Uarporn Nopmongcol ${ }^{1}$, and Tanarit Sakulyanotvittaya ${ }^{1}$

[1] $\{$ ENVIRON International Corporation, Novato, California, USA $\}$

Correspondence to: G. Yarwood (gyarwood@environcorp.com)

Supplemental Information 
Table S1. Statistical evaluation for January in 22 cities of using Equation 3 to predict ozone with 100\% NOx and VOC, 25\% NOx (100\% VOC) and zero NOx and VOC anthropogenic emissions.

\begin{tabular}{lccccccc}
\hline City & Monitors & \multicolumn{2}{c}{$100 \%$ Emissions } & \multicolumn{2}{c}{$25 \%$ Emissions } & \multicolumn{2}{c}{ Zero Emissions } \\
& & Bias $(\mathrm{ppb})$ & Error $(\mathrm{ppb})$ & Bias $(\mathrm{ppb})$ & Error (ppb) & Bias (ppb) & Error (ppb) \\
\hline Atlanta & 12 & -0.38 & 2.05 & 0.21 & 0.98 & 0.61 & 0.61 \\
Birmingham & 10 & -0.71 & 1.64 & 0.31 & 0.56 & 0.32 & 0.32 \\
Boston & 20 & 0.20 & 2.54 & 1.53 & 2.60 & 0.86 & 0.86 \\
Chicago & 25 & 0.66 & 2.06 & -0.01 & 1.45 & 0.83 & 0.89 \\
Cleveland & 11 & -0.63 & 1.95 & 0.58 & 1.09 & 0.66 & 0.66 \\
Dallas & 17 & -0.07 & 1.36 & -0.10 & 0.49 & 0.21 & 0.22 \\
Denver & 11 & 0.28 & 1.18 & -0.22 & 0.71 & 0.34 & 0.36 \\
Detroit & 11 & 0.50 & 2.24 & 0.25 & 1.77 & 0.84 & 0.87 \\
Houston & 21 & 0.40 & 2.00 & -0.34 & 0.93 & 0.72 & 1.00 \\
Los Angeles & 43 & 0.43 & 1.63 & -0.54 & 1.10 & 1.01 & 1.23 \\
Minneapolis & 8 & 0.01 & 1.23 & 0.18 & 0.80 & 0.32 & 0.33 \\
New York & 28 & 0.38 & 2.60 & 0.23 & 2.45 & 1.25 & 1.46 \\
Philadelphia & 16 & 0.59 & 2.42 & -0.26 & 2.05 & 1.21 & 1.21 \\
Phoenix & 13 & 0.72 & 2.13 & -0.36 & 1.26 & 0.69 & 0.70 \\
Pittsburgh & 14 & -0.54 & 1.97 & 0.54 & 1.20 & 0.71 & 0.71 \\
Sacramento & 14 & 0.67 & 2.22 & -0.10 & 1.05 & 0.48 & 0.49 \\
Salt Lake City & 11 & -0.03 & 1.07 & 0.03 & 0.35 & 0.26 & 0.28 \\
San Antonio & 3 & -0.44 & 0.90 & 0.09 & 0.25 & 0.11 & 0.13 \\
San Francisco & 22 & 0.44 & 1.90 & -0.21 & 0.87 & 0.54 & 0.55 \\
Seattle & 9 & -0.11 & 0.24 & 0.04 & 0.14 & 0.08 & 0.09 \\
St Louis & 15 & -0.16 & 1.65 & 0.23 & 0.84 & 0.39 & 0.40 \\
Washington DC & 27 & -0.15 & 2.59 & 0.63 & 1.70 & 0.84 & 0.84 \\
\hline All Cities & 361 & 0.19 & 1.93 & 0.09 & 1.27 & 0.71 & 0.78 \\
\hline & & & & & & & \\
\hline
\end{tabular}


Table S2. Statistical evaluation for July in 22 cities of using Equation 3 to predict ozone with 100\% NOx and VOC, 25\% NOx (100\% VOC) and zero NOx and VOC anthropogenic emissions.

\begin{tabular}{lccccccc}
\hline City & Monitors & \multicolumn{2}{c}{$100 \%$ Emissions } & \multicolumn{2}{c}{$25 \%$ Emissions } & \multicolumn{2}{c}{ Zero Emissions } \\
& & Bias (ppb) & Error (ppb) & Bias (ppb) & Error (ppb) & Bias (ppb) & Error (ppb) \\
\hline Atlanta & 12 & -2.51 & 2.62 & 0.52 & 0.56 & 0.27 & 0.30 \\
Birmingham & 10 & -1.91 & 1.92 & 0.39 & 0.40 & 0.15 & 0.18 \\
Boston & 20 & -1.85 & 1.91 & 0.18 & 0.33 & 0.38 & 0.39 \\
Chicago & 25 & -1.18 & 3.22 & 0.25 & 1.07 & 0.80 & 0.81 \\
Cleveland & 11 & -2.11 & 2.21 & 0.35 & 0.60 & 0.43 & 0.44 \\
Dallas & 17 & -1.25 & 1.43 & 0.28 & 0.33 & 0.24 & 0.25 \\
Denver & 11 & -1.45 & 2.40 & 0.56 & 0.91 & 0.47 & 0.50 \\
Detroit & 11 & -1.37 & 2.65 & 0.30 & 0.77 & 0.46 & 0.48 \\
Houston & 21 & -0.93 & 2.67 & 0.24 & 1.13 & 1.15 & 1.34 \\
Los Angeles & 43 & -3.09 & 4.12 & 1.21 & 1.85 & 2.02 & 2.06 \\
Minneapolis & 8 & -0.77 & 1.49 & 0.12 & 0.42 & 0.21 & 0.23 \\
New York & 28 & -2.17 & 2.74 & 0.24 & 0.81 & 0.78 & 0.79 \\
Philadelphia & 16 & -2.09 & 3.39 & 0.40 & 0.87 & 0.71 & 0.71 \\
Phoenix & 13 & -0.92 & 2.81 & 0.68 & 0.91 & 0.29 & 0.30 \\
Pittsburgh & 14 & -1.93 & 2.21 & 0.20 & 0.34 & 0.31 & 0.32 \\
Sacramento & 14 & -1.37 & 1.41 & 0.24 & 0.32 & 0.31 & 0.32 \\
Salt Lake City & 11 & -1.08 & 1.12 & 0.40 & 0.42 & 0.18 & 0.20 \\
San Antonio & 3 & -0.81 & 0.82 & 0.15 & 0.18 & 0.13 & 0.14 \\
San Francisco & 22 & -1.16 & 1.42 & 0.25 & 0.47 & 0.67 & 0.69 \\
Seattle & 9 & -0.98 & 1.05 & 0.07 & 0.42 & 0.20 & 0.22 \\
St Louis & 15 & -1.37 & 2.31 & 0.36 & 0.62 & 0.30 & 0.32 \\
Washington DC & 27 & -2.50 & 2.70 & 0.45 & 0.56 & 0.42 & 0.43 \\
\hline All Cities & 361 & -1.77 & 2.49 & 0.42 & 0.78 & 0.68 & 0.70 \\
\hline & & & & & & & \\
\hline
\end{tabular}


a) January, zero emissions

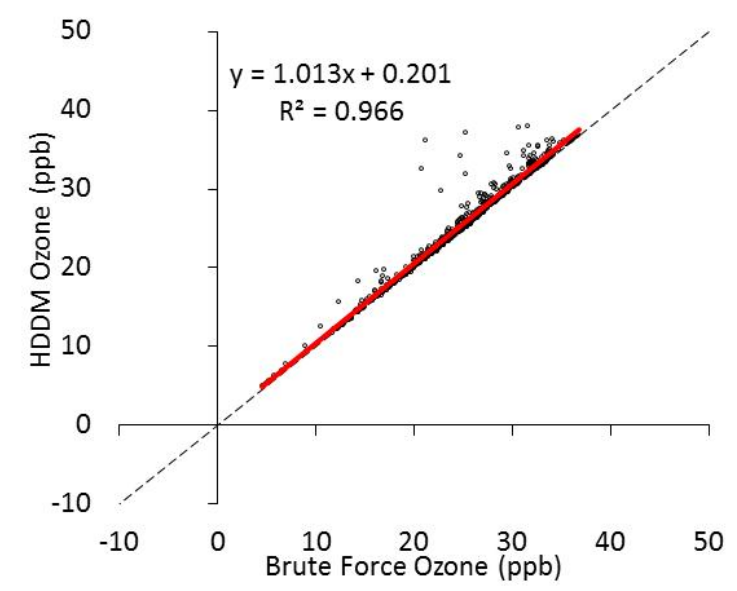

c) January, $25 \%$ emissions

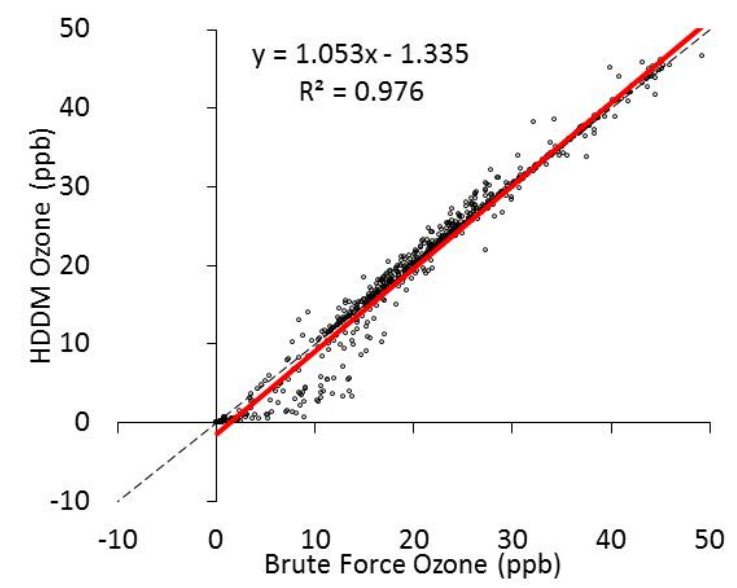

c) January, $100 \%$ emissions

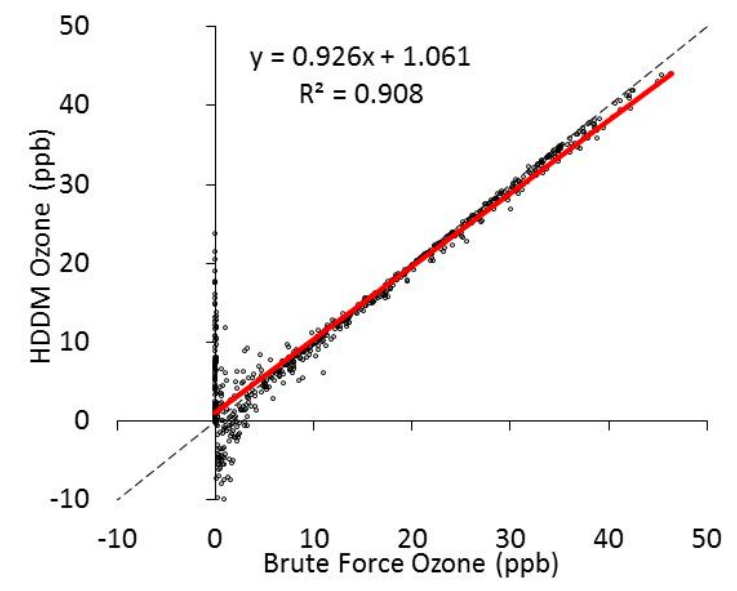

b) July, zero emissions

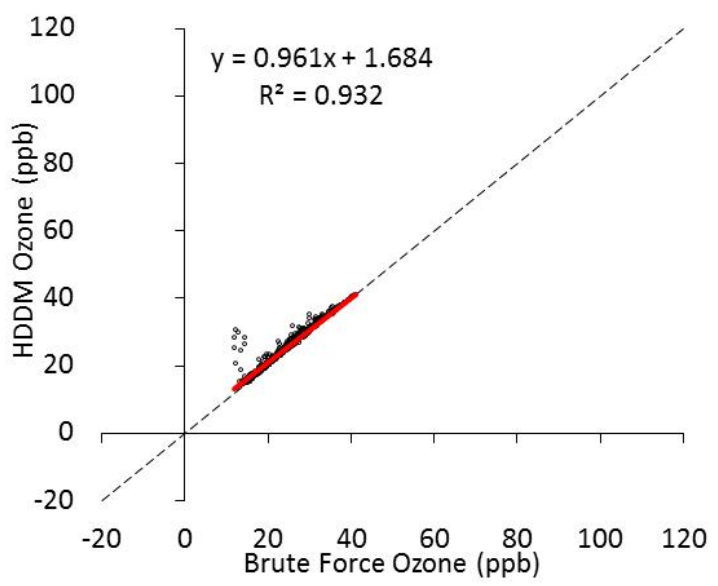

d) July, $25 \%$ emissions

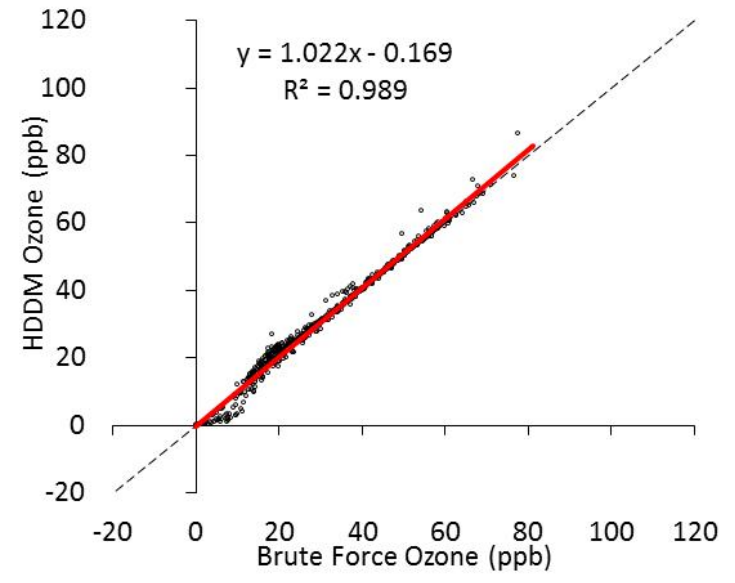

d) July, $100 \%$ emissions

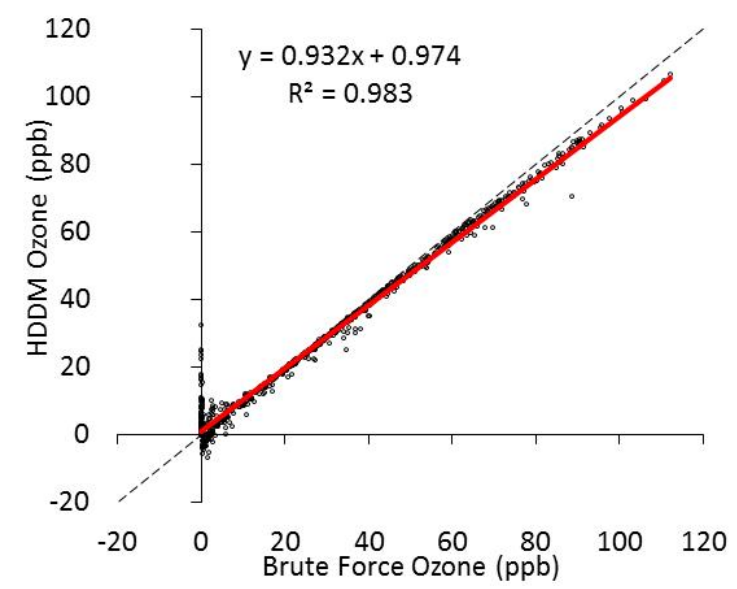


Figure S1. Hourly ozone at an urban Houston location (6400 Bissonnet Street) predicted by HDDM with Equation 3 vs. brute force CAMx simulations for: (a) zero anthropogenic NOx and VOC emissions in January; (b) zero anthropogenic NOx and VOC emissions in July; (c) $25 \%$ anthropogenic NOx (100\% VOC) emissions in January; (d) 25\% anthropogenic NOx (100\% VOC) emissions in July; (e) 100\% anthropogenic NOx and VOC emissions in January; (f) $100 \%$ anthropogenic NOx and VOC emissions in July. Dashed lines show 1:1 agreement and solid red lines show least-squares regressions. 
a) January, zero emissions

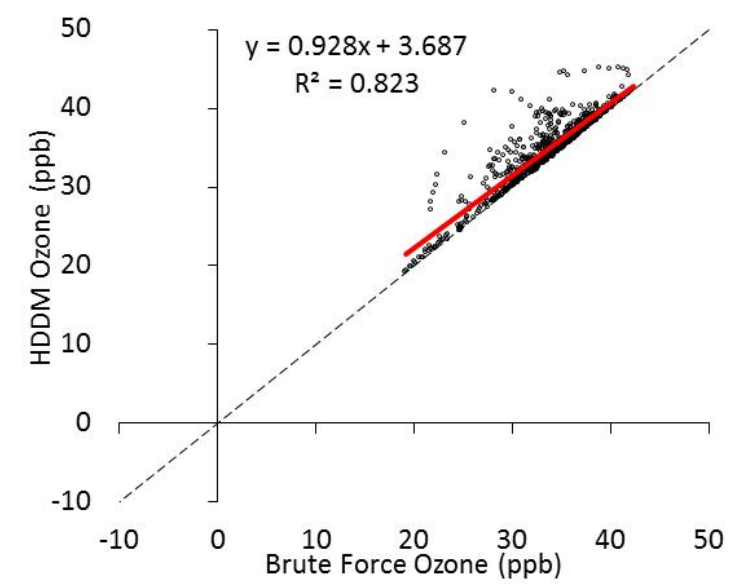

c) January, $25 \%$ emissions

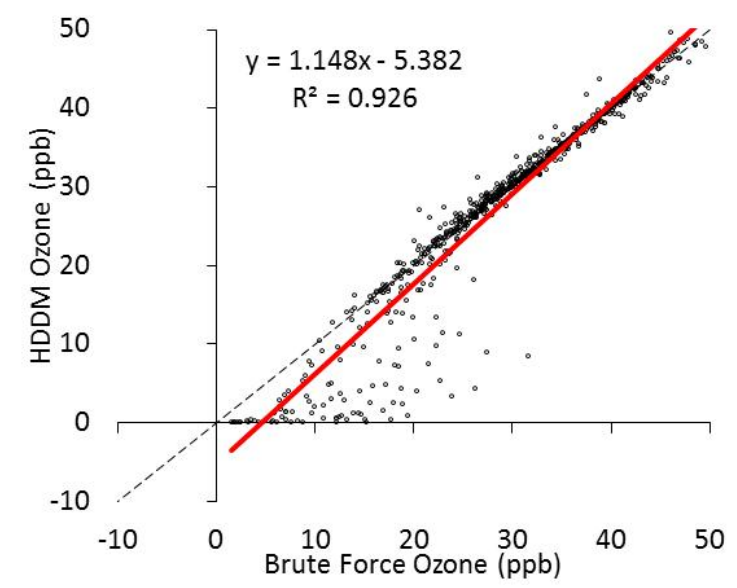

c) January, $100 \%$ emissions

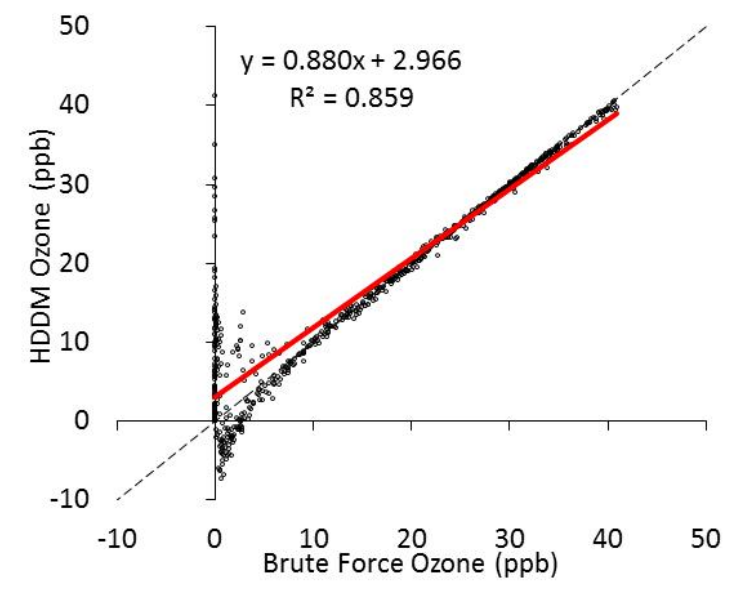

b) July, zero emissions

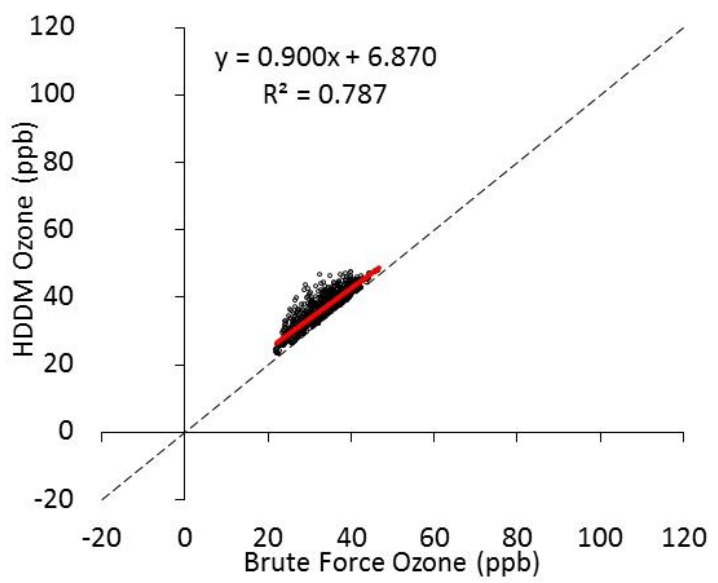

d) July, $25 \%$ emissions

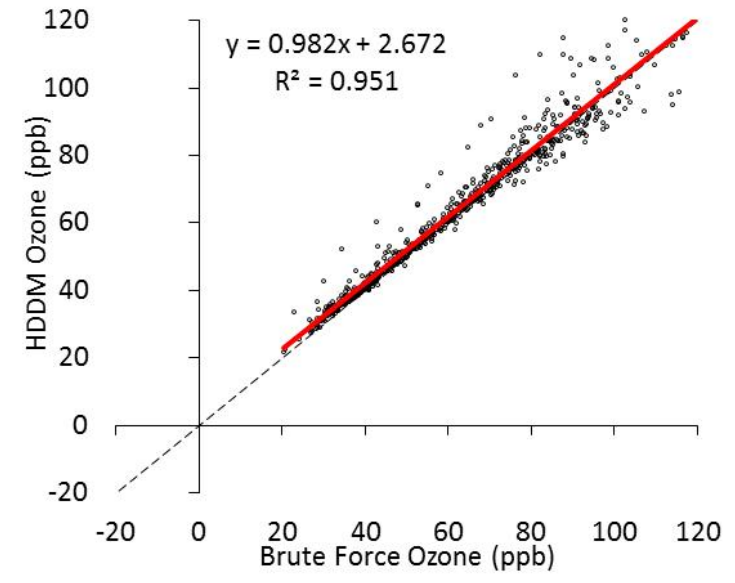

d) July, $100 \%$ emissions

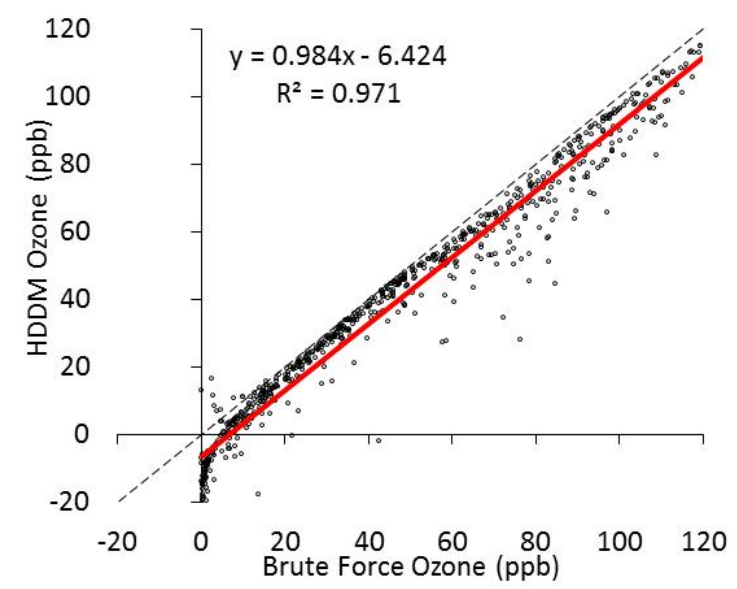


Figure S2. Hourly ozone at an urban Los Angeles location (24302 $4^{\text {th }}$ Street, San Bernardino) predicted by HDDM with Equation 3 vs. brute force CAMx simulations for: (a) zero anthropogenic NOx and VOC emissions in January; (b) zero anthropogenic NOx and VOC emissions in July; (c) 25\% anthropogenic NOx (100\% VOC) emissions in January; (d) $25 \%$ anthropogenic NOx (100\% VOC) emissions in July; (e) 100\% anthropogenic NOx and VOC emissions in January; (f) 100\% anthropogenic NOx and VOC emissions in July. Dashed lines show 1:1 agreement and solid red lines show least-squares regressions. 